\title{
Gapped Bilayer Graphene: A Tunable Strongly Correlated Band Insulator
}

\author{
V. V. Cheianov, ${ }^{1}$ I. L. Aleiner, ${ }^{2,1}$ and V. I. Fal'ko ${ }^{1}$ \\ ${ }^{1}$ Physics Department, Lancaster University, Lancaster LA1 4YB, United Kingdom \\ ${ }^{2}$ Physics Department, Columbia University, New York, New York 10027, USA
}

(Received 24 July 2011; published 7 September 2012)

\begin{abstract}
We introduce the notion of the strongly correlated band insulator (SCI), where the lowest energy excitations are collective modes (excitons) rather than the single particles. We construct controllable $1 / N$ expansion for SCI to describe their observable properties. A remarkable example of the SCI is bilayer graphene which is shown to be tunable between the SCI and usual weak coupling regime.
\end{abstract}

DOI: 10.1103/PhysRevLett.109.106801

Introduction.-Idealized models of band insulators [1] are based upon determining the spectrum of the single particle excitations (SPEs), Fig. 1(a) (I), which are characterized by the fermionic statistics, momentum $k$, charge $\pm e$, spin $1 / 2$. All the other excitations, i.e., electron-hole pairs are combined from the SPEs forming the particlehole continuum [shaded region on Fig. 1(a) (II)].

Weak electron-electron repulsion does not change the gapped spectrum of the SPEs significantly; however, it opens the decay channel of the SPE into a three-particle continuum. This process is allowed only for the particles with the energy $\epsilon$ above the threshold, see Fig. 1(b) (I). The qualitative difference appears in the two-particle spectrum: electron-hole bound states (excitons) [2] are split down from the particle-hole continuum Fig. 1(b) (II). Those discrete branches $X_{n}(k)$ [number of branches is infinite for the interaction potentials $\lim _{r \rightarrow \infty} U(r) r^{2}=\infty$ ] can not decay unless their energies exceed some threshold. We will refer to this situation as a weak-coupling insulator. All the thermodynamic and transport properties of such an insulator are described by the SPE whereas excitons are responsible for the fine structure of the optical spectra.

With the increase of the interaction the excitation hierarchy in the band insulator changes qualitatively, see Fig. 1(c). In this case, $u>X_{0}(0)$, and all the low temperature thermodynamics and the energy transport is contributed mostly by the excitons whereas the charge transport is determined by the SPEs. This leads to the different temperature dependence for the electric and thermal conductivities. Moreover, unlike in the case of the weak coupling insulators, the SPEs have a very narrow stability range [Fig. 1(c) (I)], above which the electron starts producing excitons similarly to well-known Schwinger mechanism [3] of vacuum polarization. For the same reason, only a finite number of the exciton branches are stable-all the others can decay into the two-exciton continuum [Fig. 1(c) (II)]. We will call such a system a strongly correlated insulator (SCI).

In this Letter, we present the case study of the SCI using bilayer graphene (BLG) in a transverse electric field [4-6] as an example. A strong motivation for studying BLG in
PACS numbers: 73.20.Mf, 68.65.Pq, 73.61.Ng, 78.67.-n

this context is the possibility to tune it from the weak coupling to the SCI, as it is discussed below. Using the number of electron species, $N=4$, (twofold valley and spin degeneracies) as a large parameter we obtain analytic results for observables determined by one- and twoparticle excitations. We predict that the SCI will be manifest in (a) the characteristic angle-resolved photoemission spectroscopy (ARPES) line shape with the intensity of the quasiparticle peak scaling as a nonanalytic function $\left(E / E_{1}\right)^{E_{2} / E}$ of the transverse electric field $E$, where $E_{1,2}$ are constants, and (b) the optical absorption spectrum showing exciton lines corresponding to the logarithmic attractive potential between the electron and the hole.

Model.-The band structure of BLG is described by the effective single-particle Hamiltonian [7] $(\hbar=1)$
(I)

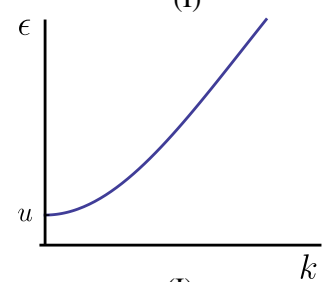

(I)

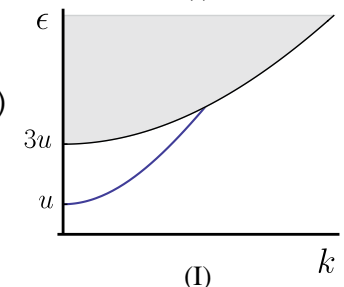

(I)

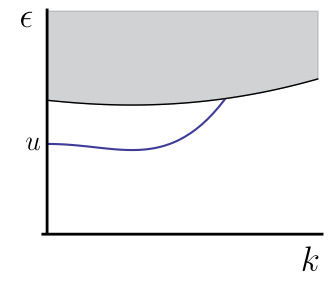

(II)

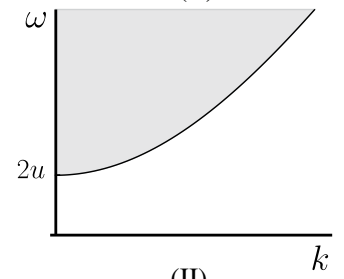

(II)

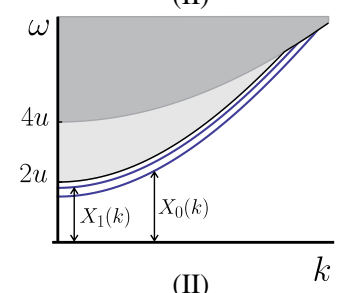

(II)

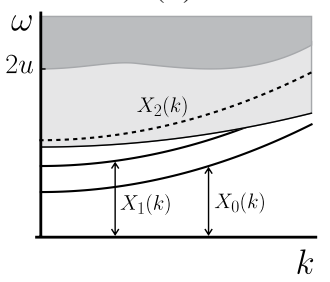

FIG. 1 (color online). One- (I) and two-particle spectra (II) in the insulator. (a) Noninteracting particles; (b) the weak coupling; (c) SCI; Particle-hole symmetry is implied for simplicity. 


$$
\hat{H}_{0}=\left(\begin{array}{cc}
u_{0} & \left(k_{x}+i k_{y}\right)^{2} /(2 m) \\
\left(k_{x}-i k_{y}\right)^{2} /(2 m) & -u_{0}
\end{array}\right) \text {, }
$$

where $u_{0} \propto E$ is the interlayer asymmetry tunable by the transverse electric field $E, m$ is the effective mass, and $\mathbf{p}=\left(p_{x}, p_{y}\right)$ is the Bloch momentum counted from the $K$ point of the Brillouin zone. Excitations whose momenta are near $K^{\prime}$ are described by the parity conjugate of the Hamiltonian (1) and form another particle species with identical properties. The fourfold degenerate spectrum of the SPEs corresponding to Eq. (1) is

$$
\epsilon(k)=\left[u_{0}^{2}+k^{4} /\left(4 m^{2}\right)\right]^{1 / 2} \approx u_{0}+k^{4} /\left(8 u_{0} m^{2}\right) .
$$

We will see that SCI behavior of BLG is associated with the long range part of the Coulomb interaction $v(\mathbf{r})=e^{2} / r$ so that the effective Hamiltonian is

$H=\int d^{2} r \psi^{\dagger} \hat{H}_{0} \psi+\frac{1}{2} \int d^{2} r d^{2} r^{\prime} \rho(\mathbf{r}) v\left(\mathbf{r}-\mathbf{r}^{\prime}\right) \rho\left(\mathbf{r}^{\prime}\right)$.

We omitted here the short range interaction with nontrivial matrix structure. Those terms are related to the symmetry breaking in BLG and get renormalized with increasing the linear scale [8]. All those renormalizations, as well as the renormalization of the electron mass and interlayer asymmetry, stop at distances of the order of

$$
\ell=1 / \sqrt{m u_{0}},
$$

and are incorporated into the SPE spectrum (1). In Eq. (3), $\hat{H}_{0}$ given in Eq. (1) operates on a two-component vector $\psi(\mathbf{r})=\left[\psi_{A}(\mathbf{r}), \psi_{B}(\mathbf{r})\right]^{T}$ in the sublattice space (summation over the spin and valley indices is implied), $\rho=$ : $\psi^{\dagger} \psi$ : is the normal-ordered particle density.

Dimensional analysis of the Hamiltonian (3) reveals only one dimensionless coupling constant

$$
\kappa^{2}=m e^{4} / u_{0},
$$

which diverges as $u_{0} \rightarrow 0$ signaling the SCI. In this limit perturbation theory in $e^{2}$ fails; however, the theory remains amendable to the $1 / N$ expansion which uses the dynamically screened Coulomb interaction as the leading-order approximation (see the Supplemental Material [9]for the formal discussion).

Screened interaction in large $N$ approximation is obtained as a resummation of Fig. 2(a):

$$
V(q, i \omega)=v(q)[1+N v(q) \Pi(q, i \omega)]^{-1},
$$

where $v(q)=2 \pi e^{2} / q$ and $\Pi(q, i \omega)$ is the polarization function in the momentum-frequency domain with asymptotic expressions shown in Fig. 2(b). Taking $N \gg 1$ limit, one finds $\kappa$ to drop out of the expression giving

$$
V(q, i \omega) \simeq 1 /[N \Pi(q, i \omega)] .
$$

Thus, the perturbation series becomes an expansion in powers of $1 / N$. Such an expansion, however, contains (a)
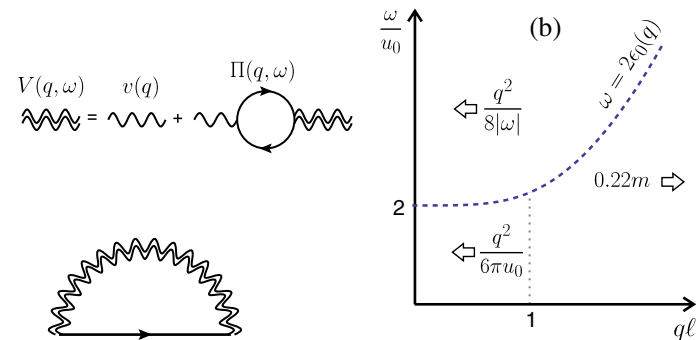

(c)

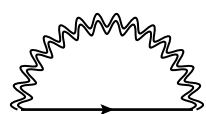

(d)

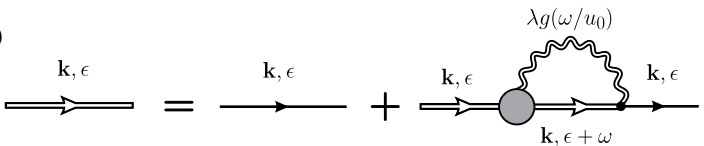

(e)

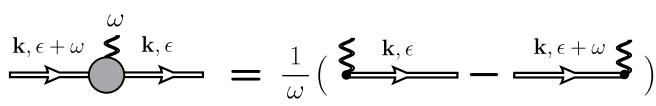

FIG. 2 (color online). (a) Resummation of the leading $N$ loops leads to the RPA screening of the interaction potential. (b) Asymptotic regions for the polarization operator. (c) The leading-order self energy correction. (d) Resummation of the leading logarithm using Ward-Takahashi identity (e).

singular terms caused by $\Pi(q \rightarrow 0, i \omega) \rightarrow 0$. The expression for the screened static potential is most instructive:

$$
V(r) \equiv \int \frac{d^{2} q e^{i \mathbf{q r}}}{(2 \pi)^{2}} V(\mathbf{q}, 0)=\left\{\begin{array}{ll}
\frac{3 u_{0}}{N} \ln \frac{R_{*}}{r}, & r \ll R_{*} \\
\frac{e^{2}}{r}, & r \gg R_{*}
\end{array},\right.
$$

where a new spatial scale appeared:

$$
R_{*}=\kappa N \ell \text {. }
$$

Notice that $V(\ell)>u_{0}$ at small enough $u_{0}$, which makes the SCI insulator regime possible. At larger $u_{0}$, interaction is weak and may be treated perturbatively.

SPE spectrum and particle residue.-We start with calculating the leading order in $1 / N$ self-energy correction, Fig. 2(c). Finding the pole of the resulting Green function, we obtain instead of Eq. (2)

$$
\epsilon(k)=u-\frac{0.680}{N} \frac{k^{2}}{2 m}+\frac{k^{4}}{8 m^{2} u_{0}}[1+\mathcal{O}(1 / N)] .
$$

The single particle gap is strongly enhanced:

$$
u=u_{0}\left(1+\frac{3}{2 N} \ln \kappa N\right) .
$$

In the limit of $\kappa \rightarrow \infty$ this enhancement diverges which, as we will see shortly, signals the transition to the SCI. The physical meaning of gap renormalization is that the band insulator can not completely readjust itself when a charged particle is introduced. The interaction of the extra particle with the dipoles from the distances between $\ell$ and $R_{*}$ leads to the logarithmic divergence for $R_{*} \rightarrow \infty$. The second term in Eq. (10) is the interaction-induced negative curvature analytic in $1 / N$. It significantly exceeds contribution of 
Ref. [7] to the resulting "mexican hat" spectrum shown on Fig. 1(c) (I).

The single-particle residue found from the same diagram of Fig. 2(c) is given by

$$
Z \approx 1-\left(8 / \pi^{2} N\right) \ln (\kappa N) \ln \left(\Lambda / u_{0}\right),
$$

where $\Lambda \approx 0.2 \mathrm{eV}$ is the upper limit for the applicability of the two-band model (1).

Equations (11) and (12) contain logarithmically divergent factors, which makes it necessary to sum up all orders of the perturbation theory in

$$
\lambda \equiv(\ln \kappa N) /(2 \pi N) .
$$

The summation procedure outlined below shows that the gap (11) does not acquire any higher order in $\lambda$ corrections whereas Eq. (12) changes to

$$
Z=\exp \left[-\left(8 / \pi^{2} N\right) \ln (\kappa N) \ln \left(\Lambda / u_{0}\right)\right] .
$$

This result is consistent with the renormalization group equations obtained earlier in Ref. [10], where the renormalization flow is stopped at the energy scale $u_{0}$.

Let us sketch derivations of Eqs. (11) and (14). We notice that the dominant logarithmic divergence is contributed by the momenta transfer $q \ell \lesssim 1$ through any interaction propagator. This allows for following simplifications: (i) to factorize the polarization operator as

$$
\Pi(q, i \omega)=q^{2} /\left[u_{0} g\left(i \omega / u_{0}\right)\right],
$$

where $g(z)$ is a function well defined in the limit $\kappa \rightarrow \infty$; (ii) to neglect the change in the momentum $k$ of any single particle Green function (GF). The latter allows the integration over the momenta in each interaction line separately: the screened interaction (6) is replaced with

$$
\int^{q \ell \leq 1} V(q, i \omega) d^{2} q /(2 \pi)^{2}=u_{0} \lambda g\left(i \omega / u_{0}\right) .
$$

A nontrivial aspect of Eq. (15) is the appearance on the bare scale $u_{0}$ rather than the renormalized gap $u$ in the right-hand-side of the equation. The reason for this is that divergence $\propto \ln N \kappa$ can not enter into $\Pi$, because the screening is determined by the neutral dipoles [11].

The single-particle line shape in angle-resolved photoemission spectroscopy.-The suppression of the singleparticle residue in Eq. (14) suggests that the ARPES spectrum of the SCI in the vicinity of the band gap is strongly affected by the electron-electron correlations. In order to find the exact line shape we close the Dyson equation for the strong coupling theory, Fig. 2(d), using the Ward-Takahashi identity, Fig. 2(e). This results in an exact time ordered GF

$$
\begin{aligned}
G(\mathbf{k}, \epsilon) & =\int_{-\infty}^{\infty} d t e^{i \epsilon t} G_{0}(\mathbf{k}, t) e^{-(\lambda / 2) F(t)} \\
F(t) & =i g(0) u|t|+\int_{2}^{\Lambda / u_{0}} d z\left(1-e^{-i z|t|}\right) W(z),
\end{aligned}
$$

where $G_{0}(\mathbf{k}, t)$ is the bare GF in the momentum-time domain, and $W(z) \equiv i[g(z+i 0)-g(z-i 0)] /\left(\pi z^{2}\right)$. Expression (17) is valid for any function $g$. For the polarization loop neglecting vertex corrections and renormalization of the GF, $N \rightarrow \infty$ [11], we find $g(0)=6 \pi$ and

$$
W(z)=\frac{16 \pi z}{z^{2}+4}\left[\pi^{2}+\left(\ln \frac{z+2}{z-2}-\frac{4 z}{z^{2}+4}\right)^{2}\right]^{-1} .
$$

Then, $F(t)=6 \pi i u|t|+(16 / \pi) \ln \left(\Lambda / u_{0}\right)$ and Eq. (17) leads to Eqs. (11) and (14).

Equations (17) enables one to calculate not only the particle pole but also the incoherent contribution describing the coupling of the extra electron introduced or extracted from the system (as in the tunneling or photoemission experiments) with a many particle continuum. To do so, we evaluate $A(\epsilon)=(1 / \pi)|\operatorname{Im} \operatorname{Tr} G(0, \epsilon)|$ by numerical integration of Eq. (17). [Due to the electron-hole symmetry, $A(\epsilon)=A(-\epsilon)$, and the structure and finite $k$ is similar]. The result plotted in Fig. 3 shows the single particle peak at $\epsilon=u \gg u_{0}$ and the threshold at $\epsilon=u+2 u_{0}$ due to the coupling to the three-particle continuum [12].

Exciton spectra.-Next we discuss the collective spectrum of the system, revealed as the poles of two-particle propagators of the system. To calculate those poles, $X$, in leading $1 / N$ approximation, it is sufficient to neglect the retardation in the interaction potential (6) and consider Schrödinger's equation for an electron and a hole,

$\left\{\frac{\hat{p}_{e}^{4}+p_{h}^{4}}{8 m^{2} u_{0}}+[2 u-V(r)]\right\} \Psi\left(r_{e}, r_{h}\right)=X \Psi\left(r_{e}, r_{h}\right)$,

with $V(r)$ defined in Eq. (8), and $\mathbf{r} \equiv \mathbf{r}_{e}-\mathbf{r}_{h}$. Unbound states with $X>2 u$ correspond to the particle-hole continuum and the bound states are the exciton lines.

Due to the nonparabolicity of the one-particle spectrum, the motion of the exciton center of mass $\mathbf{P}=\mathbf{p}_{e}+\mathbf{p}_{h}$ can not be separated from the relative motion, and we consider

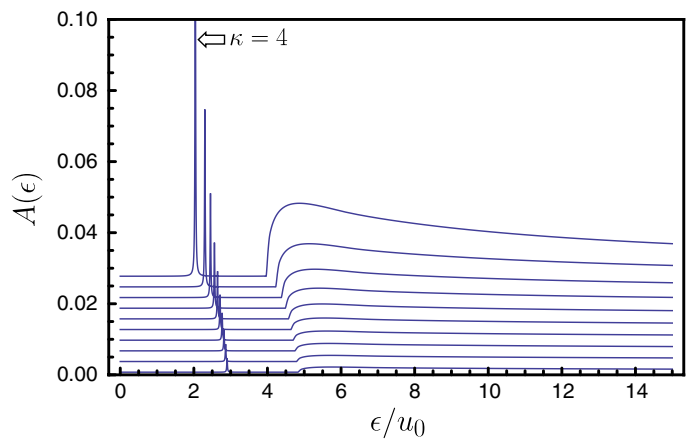

FIG. 3 (color online). The energy-dependent electron spectral weight $A(\epsilon)$, representing the ARPES line shape near the $K$ point of the Brillouin zone of BLG at different values of the coupling constant $\kappa$. For clarity, the curves corresponding to ten equidistant values of $\kappa=4,8,12, \ldots, 40$ are vertically offset and the quasiparticle peak is artificially broadened and scaled down by a factor of 100 . 


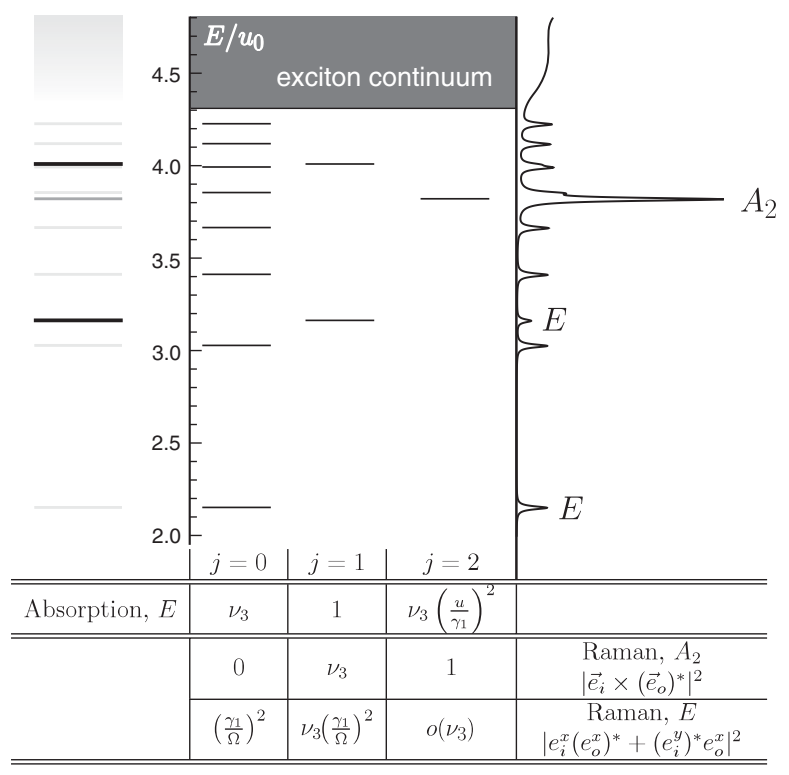

FIG. 4. The energy levels of the exciton in units of $u_{0}$ for $N=4$. Hatched region is the two-exciton continuum. Side panels are the sketches of the absorption (left) and Raman spectrum (right). Bottom table contains the oscillator strengths of the transitions in units of the strongest line, $\vec{e}_{i, o}$ are the complex polarization vectors for the incoming (i) and outgoing (o) light, $\Omega$ is the base line frequency, and $\gamma_{1} \simeq 0.2 \mathrm{eV}$. The coefficient $\nu_{3} \simeq\left(\mathcal{E}_{\mathrm{LiTr}} / u\right)^{2}$ is not zero only due to the trigonal warping of the spectrum, where $\mathcal{E}_{\mathrm{LiTr}} \simeq 1 \mathrm{meV}$ is the energy of the Lifshitz transition in gapless, $u_{0}=0$, BLG $[7,8]$.

here the case of $P=0$ relevant for optics. In this case, the levels are labeled by the normal angular momenta $|j|=0,1,2, \ldots$ and by the radial quantum number $n=0,1, \ldots$. Dimensional analysis gives the low lying states with the size of the order of $\ell \ll R_{*}$; thus, $[2 u-V(r)]=u_{0}[2+(3 / 2 N) \ln r / \ell]$ does not depend on $\kappa \gg 1$, and, for $n+|j| \lesssim N \kappa$,

$$
X_{n}^{j}=u_{0}\left[2+\frac{3}{N}\left(\frac{1}{4} \ln \frac{12}{N}+\xi_{n}^{j}\right)\right]
$$

where $\xi_{n}^{j}$ are the eigenvalues of the differential operators

$$
\hat{h}^{j}=\left(-d^{2} / d z^{2}-z^{-1} d / d z+j^{2} / z^{2}\right)^{2}+\ln z .
$$

Results of the numerical diagonalization of $\hat{h}^{j}$ are plotted on Fig. 4. Note that the levels with $X_{n}^{j}>2 X_{0}^{0}$ decay into a two exciton continuum as in Fig. 1(c) (II).

Since the spectrum of the excitons (19a) does not depend on the coupling constant $\kappa$, the system can be quite easily tuned in the SCI regime, $u \gg X_{0}^{0}$.

The fact that energies of the lowest-lying excitons in Eq. (19a) scale with the bare gap parameter $u_{0}$ rather than the renormalized single-particle gap $u$ justifies the scaling form of the polarization operator Eq. (15). Indeed, for the strong coupling regime, the polarization of BLG occurs via the appearance of virtual excitons with $j= \pm 1$, so that
$\Pi\left(q \ll \ell^{-1}, \omega\right)$ is generic for both the weak coupling regime and SCI. Moreover, $g(0)$ acquires only $1 / N$ corrections, so that Eq. (11) does not change. The fine structure of $g(z)$ shows the exciton resonances, it changes the threshold in $A(\epsilon)$ by the exciton binding energy $\simeq u_{0} / N$, and introduces an additional fine structure which may be distinguished in higher derivatives of $A(\epsilon)$

Exciton lines in SCI optics.-The exciton lines (19a) are degenerate $\left(N^{2}\right.$ for $j=0$, and $2 N^{2}$ for $\left.|j|>0\right)$ due to the $N$-fold degeneracy of the electron spectrum. Such degeneracy is lifted due to the crystalline symmetry [13].

First, the fourfold spin degeneracy is split to the $S=0$ singlet and $S=1$ triplet states due to the exchange interaction. In the absence of spin-orbit interaction, triplet excitons cannot be observed in optical experiments. Spinsinglet states are further split due to the trigonal symmetry of the bilayer crystal and should be classified according to the irreducible representations of its planar group, only $A_{1,2}$ and $E$ representations are optically active. Such a classification is presented in Fig. 4 together with the selection rules $\left(A_{1}\right.$ is not active in Raman because of the electronhole symmetry). These rules for the bright $E$-exciton absorption (as well as luminescence), $\sigma^{ \pm} \rightarrow X^{1} \rightarrow \sigma^{ \pm}$are determined by the form of the interband current operator derived from the Hamiltonian Eq. (1), and a trigonal warping term [7] due to skew interlayer hopping, for a weak transition $\sigma^{ \pm} \rightarrow X^{0}$. The selection rules for Raman processes are determined by the electron-two photon interaction via a virtual intermediate state [14], with the dominant transition $A_{2}: \sigma^{ \pm} \rightarrow \pm i \sigma^{ \pm}+X^{2}$ and a satellite $E: \sigma^{ \pm} \rightarrow \sigma^{\mp}+X^{0}$.

Conclusion.-We presented a general and controllable theory of a SCI: a band insulator where the spectrum of excitons lies deep below the lowest branch of the singleparticle spectrum. Gapped bilayer graphene is not the only example with such properties, the list of other potential SCIs includes quantum wells of semimetal compounds, such as $\mathrm{Bi}_{1-x} \mathrm{Sb}_{x}$, or silicene [15] in a transverse electric field [16]. However, BLG is unique in its tunability from the weak coupling to the SCI. In the lab, this tuning can be achieved by the application of the electric field normal to the BLG plane.

We thank L.I. Glazman for useful discussions. The work was supported by the European Research Council through the ERC Advanced Grant (V.F.) and the ERC Starring Grant (V.C.); and by the Simons Research Foundation (I. A.).

[1] See, e.g., C. Kittel, Introduction to Solid State Physics (Wiley Press, New York, 2005), 8th ed.

[2] G. Wannier, Phys. Rev. 52, 191 (1937); N. F. Mott, Trans. Faraday Soc. 34, 500 (1938).

[3] J. S. Schwinger, Phys. Rev. 82, 664 (1951). 
[4] K. S. Novoselov, E. McCann, S. V. Morozov, V. I. Fal'ko, M. I. Katsnelson, U. Zeitler, D. Jiang, F. Schedin, and A. K. Geim, Nature Phys. 2, 177 (2006).

[5] Y. Zhang, T.-T. Tang, C. Girit, Z. Hao, M. C. Martin, A. Zettl, M. F. Crommie, Y.R. Shen, and F. Wang, Nature (London) 459, 820 (2009).

[6] R. T. Weitz, M. T. Allen, B.E. Feldman, J. Martin, and A. Yacoby, Science 330, 812 (2010).

[7] E. McCann and V. I. Fal'ko, Phys. Rev. Lett. 96, 086805 (2006).

[8] Y. Lemonik, I. L. Aleiner, C. Toke, and V. I. Fal'ko, Phys. Rev. B 82, 201408 (2010); Y. Lemonik, I. L. Aleiner, and V. I. Fal'ko, Phys. Rev. B 85, 245451 (2012).

[9] See Supplemental Material at http://link.aps.org/ supplemental/10.1103/PhysRevLett.109.106801 for a detailed discussion of the $1 / N$ expansion in bilayer graphene.

[10] R. Nandkishore and L. Levitov, Phys. Rev. B 82, 115431 (2010).

[11] We will elaborate on this after the exciton is considered.

[12] In fact $A(\epsilon)$ is singular at $\epsilon_{n}=u+2 n u_{0}$ but those additional singularities are seen only in higher derivatives.

[13] Excitons: Selected Chapters, edited by E. I. Rashba and M. D. Sturge (North-Holland, Amsterdam, 1987).

[14] M. Mucha-Kruczynski, O. Kashuba, and V.I. Fal'ko, Phys. Rev. B 82, 045405 (2010).

[15] S. Cahangirov, M. Topsakal, E. Aktürk, H. Şahin, and S. Ciraci, Phys. Rev. Lett. 102, 236804 (2009).

[16] N. D. Drummond, V. Zolyomi, and V. I. Fal'ko, Phys. Rev. B 85, 075423 (2012). 